\title{
Comparison of CHOP vs CHOPE for treatment of peripheral T-cell lymphoma: a meta-analysis
}

This article was published in the following Dove Medical Press journal: OncoTargets and Therapy

\author{
Shu Deng' \\ Shenyun Lin' \\ Jianping Shen' \\ Yuqing Zeng ${ }^{2}$ \\ 'Department of Hematology, \\ The First Affiliated Hospital of \\ Zhejiang Chinese Medicine University, \\ Hangzhou, Zhejiang 310006, China \\ ${ }^{2}$ Department of Orthopedics, TongDe \\ Hospital of Zhejiang Province, \\ Hangzhou, Zhejiang 3100I2, China
}

Correspondence: Yuqing Zeng Department of Orthopedics, TongDe Hospital of Zhejiang Province, Hangzhou, Zhejiang 310012 , China

Tel +86 57I 86620325

Email kanlao767yiyu@163.com
Objective: To compare cyclophosphamide, doxorubicin, vincristine, and prednisone (CHOP) and CHOP plus etoposide (CHOPE) with regard to outcomes including efficacy and safety for patients with peripheral T-cell lymphoma (PTCL).

Methods: Relevant literature was searched using PubMed, Embase, Wanfang, and CNKI for eligible trials comparing CHOP with CHOPE for treatment of PTCL. The following outcomes of PTCL patients were considered: complete response (CR), partial response (PR), overall response rate (ORR), and adverse events (AEs; grade $\geq 3$ ). Risk ratios (RRs) were appropriately derived from fixed-effects or random-effects models.

Results: A total of five prospective or retrospective articles with 1,560 patients were elected for the meta-analysis. There were no significant differences in CR ( $R R=1.11,95 \%$ CI: $0.73-1.67$, $P=0.632)$, PR (RR $=1.40,95 \%$ CI: $0.52-3.76, P=0.504)$, and ORR ( $R R=1.25,95 \%$ CI: $0.93-1.69$, $P=0.146)$ between the CHOP and CHOPE groups. However, AEs including anemia ( $\mathrm{RR}=1.69$, 95\% CI: $1.33-2.16, P<0.001)$ and thrombocytopenia (RR $=1.43,95 \%$ CI: $1.15-1.77, P=0.001)$ were significantly increased in CHOPE group compared to that in CHOP group.

Conclusion: Meta-analysis suggested that there were no differences in therapeutic effect for patients with PTCL between CHOP and CHOPE groups with regards to CR, PR, and ORR, whereas the CHOPE group had significantly increased AEs (anemia and thrombocytopenia) compared to CHOP group.

Keywords: peripheral T-cell lymphoma, complete response, partial response, overall response rate, adverse events

\section{Introduction}

Peripheral T-cell lymphoma (PTCL) is a highly heterogeneous malignancy accounting for $10 \%-15 \%$ of all non-Hodgkin's lymphomas in the Western world, ${ }^{1}$ and its incidence is higher in East Asia. ${ }^{2,3}$ According to the international T-Cell Lymphoma Project, the major subtypes of PTCL are composed of PTCL not otherwise specified, angioimmunoblastic T-cell lymphoma, and anaplastic large-cell lymphoma. ${ }^{4}$ Moreover, due to the varied morphology of subtypes, the classification and diagnosis of this disease has been a great challenge. So far, the optimal strategy for PTCL treatment is still unclear, and although cyclophosphamide, doxorubicin, vincristine, and prednisone (CHOP) is the standard first-line chemotherapy treatment, most patients still have a poor prognosis with median overall survival (OS) of 6.5 months because of rapid relapse. ${ }^{5,6}$ During the past years, more and more therapeutic agents with novel mechanisms of action, such as romidepsin, ${ }^{7}$ belinostat, ${ }^{8}$ brentuximab vedotin, ${ }^{9}$ and pralatrexate ${ }^{10}$ have been approved for PTCL treatment. So far, according to the guidelines of National Comprehensive Cancer Network, combination chemotherapies are regarded as the second-line therapy for patients with relapsed PTCL. ${ }^{5}$ 
Etoposide, inducing DNA double-strand breaks through the inhibition of DNA topoisomerase II activity, has been widely used as an anticancer chemotherapeutic drug. ${ }^{11,12}$ Recently, CHOP plus etoposide (CHOPE) has demonstrated survival benefit for patients with PTCL. ${ }^{13-15}$ However, CHOPE chemotherapy has yielded contradictory results, ${ }^{13,16}$ and the benefit has been limited to relatively young patients (aged less than 60 years). ${ }^{15}$ Therefore, this study aimed to assess whether CHOPE could lead to better postoperative functional recovery in terms of complete response (CR), partial response (PR), overall response rate (ORR), and adverse events (AEs) compared with CHOP.

\section{Methods}

\section{Literature search}

All studies on PTCL therapeutic regimens performed with CHOP and CHOPE were searched using several major databases including PubMed, Embase, Wanfang, and CNKI with the search terms "(peripheral t-cell lymphoma) AND (etoposide OR VP16) AND ((cyclophosphamide AND doxorubicin AND vincristine AND prednisone) OR CHOP)". No restrictions on language were applied during the retrieval, and the retrieval time was until February 1, 2018. Moreover, in order to get more literature, we performed manual retrieval of paper documents, and references in relevant reviews and included studies were screened.

\section{Selection criteria}

Studies which met the following criteria were selected: 1) clinical research with prospective or retrospective experimental design. 2) The therapeutic effect of CHOPE vs CHOP for PTCL was adopted in the trials, and the outcomes mainly included CR, PR, ORR, and AEs (grade $\geq 3$ ) including neutropenia, anemia, thrombocytopenia, leukopenia, and vomiting. Correspondingly, exclusion criteria were review articles, comments, and letters. Literature repeatedly published or used for multiple studies by the same population were excluded.

\section{Data extraction}

The relevant data were independently extracted and analyzed by two investigators, including the first author, date published, study area, participant age and gender, sample size, type of patients, research type, and the outcomes of patients. All disagreements were resolved by discussion.

\section{Statistical analysis}

All statistical analyses were performed with Stata 11.0 software, and risk ratios (RRs) with 95\% CI were calculated for dichotomous variables. Cochran's Q and I ${ }^{2}$ metrics were applied to assess the heterogeneity. ${ }^{17}$ The fixed-effect model was used if no heterogeneity existed $\left(P \geq 0.05, \mathrm{I}^{2} \leq 50 \%\right)$; otherwise, the random-effects model was chosen. In addition, Egger's test was used for assessment of publication bias, and the stability of the result was studied using sensitivity analysis. $P<0.05$ was considered statistically significant.

\section{Results}

\section{Search results}

A total of 743 articles were extracted from PubMed ( $n=77)$, Embase ( $\mathrm{n}=627)$, Wanfang $(\mathrm{n}=27)$, and CNKI $(\mathrm{n}=12)$. A total of 692 studies remained after excluding 51 duplicate articles. After screening the title of studies, 663 articles not meeting the inclusion and exclusion criteria were excluded. Then, the remaining 29 articles were further assessed by reading the abstract and full-text, leaving five articles with 1,560 patients which eventually fulfilled the selection criteria ${ }^{18-22}$ (Figure 1).

\section{Characteristics of included studies}

The basic information of these publications is given in Table 1, and according to the data, we found the included articles contained four retrospective studies ${ }^{18-21}$ and one prospective research..$^{22}$ All articles were published from 2013-2017, and among them, three trials were from China, one from Thailand, and one from Korea. Additionally, the study of Kim et al included two sets of data, one of which was from the Seoul National University Hospital and one from the Korea Central Cancer Registry. Hence, there were six groups of data in our meta-analysis with a sample size from $32-1,255$.

\section{Results of meta-analysis $C R$, $P R$, and $O R R$}

As shown in Figure 2, the pooled results of CR, PR, and ORR for PTCL patients between two groups were compared. Subsequently, five articles compared the CR of CHOP and CHOPE. ${ }^{18-22}$ Because significant heterogeneity across studies ( $P=0.040, \mathrm{I}^{2}=60.2 \%$ ) was detected, the random-effects model was applied and it revealed no significant difference of CR between CHOP and CHOPE (RR $=1.11,95 \%$ CI: 0.73-1.67, $P=0.632$, Figure $2 \mathrm{~A}$ ).

Likewise, four trials reported the PR and ORR, ${ }^{18,20,22}$ and significant heterogeneity was found between them $(P=0.045$, $\mathrm{I}^{2}=62.7 \%$; and $P=0.050, \mathrm{I}^{2}=61.5 \%$; Figure $2 \mathrm{~B}$ and $\mathrm{C}$ ); therefore, the meta-analysis with random-effects model illustrated no significant differences in PR ( $R R=1.40,95 \%$ CI: $0.52-3.76$, 


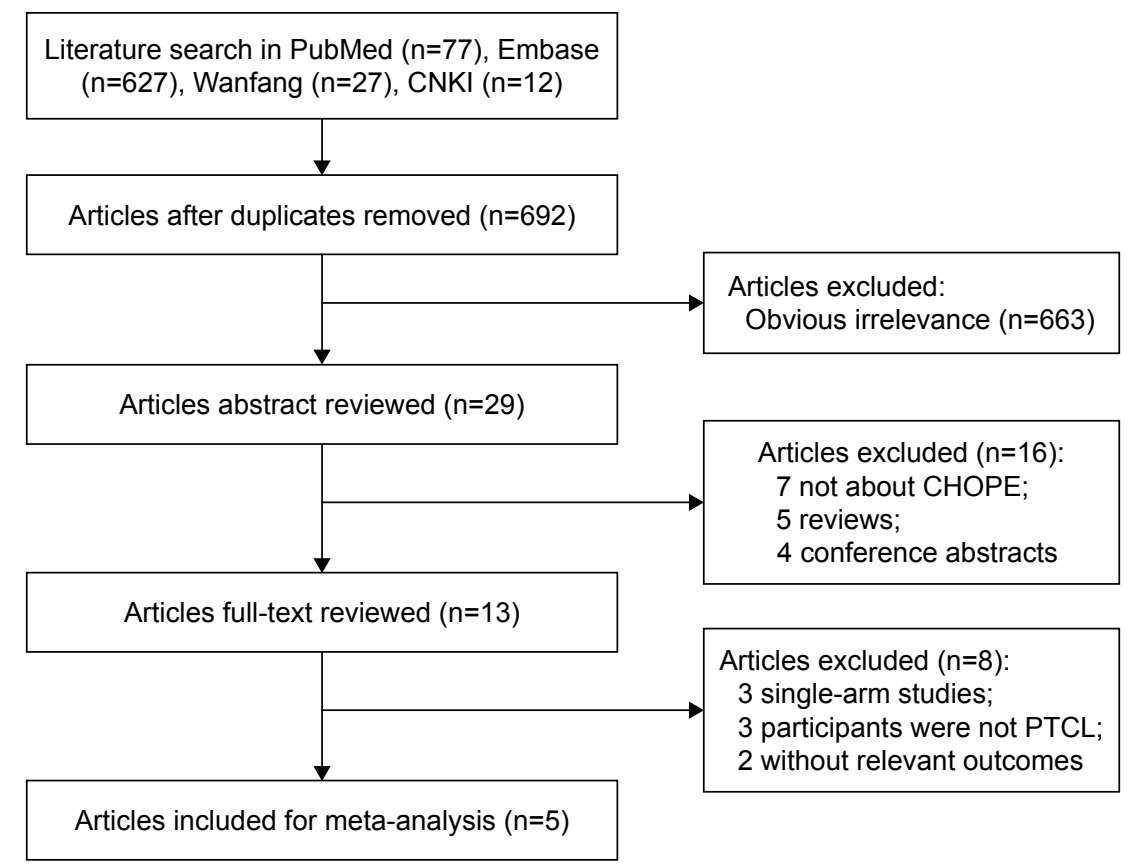

Figure I Flow chart of study literature selection for meta-analysis.

Abbreviations: CHOPE, cyclophosphamide, doxorubicin, vincristine, and prednisone plus etoposide; PTCL, peripheral T-cell lymphoma.

$P=0.504)$ and $\mathrm{ORR}(\mathrm{RR}=1.25,95 \% \mathrm{CI}: 0.93-1.69, P=0.146)$ between the two groups. Taken together, our results revealed that the differences in CR, PR, and ORR for PTCL between $\mathrm{CHOP}$ and $\mathrm{CHOPE}$ groups were not significant.

\section{Adverse events}

There were two, ${ }^{18,19}$ three, ${ }^{19,21,22}$ three, ${ }^{18,19,21}$ three, ${ }^{18,21,22}$ and two ${ }^{18,21}$ included studies that reported complications of neutropenia, anemia, thrombocytopenia, leukopenia, and vomiting, respectively. However, the results showed no significant heterogeneity for these five indicators, respectively $\left(\mathrm{I}^{2}<50 \%\right.$ and $\left.P>0.05\right)$; therefore, the fixed-effect model was applied. As illustrated in Figure 3, the differences were significant for anemia ( $\mathrm{RR}=1.69,95 \% \mathrm{CI}$ : $1.33-2.16, P<0.001$, Figure $3 \mathrm{~A})$ and thrombocytopenia ( $\mathrm{RR}=1.43,95 \% \mathrm{CI}$ : $1.15-1.77, P=0.001$, Figure $3 \mathrm{~B}$ ) between $\mathrm{CHOP}$ and $\mathrm{CHOPE}$; while no significant difference was found for neutropenia $(\mathrm{RR}=1.01,95 \% \mathrm{CI}: 0.73-1.39, P=0.958$, Figure 3C), leucopenia $(\mathrm{RR}=1.14,95 \% \mathrm{CI}: 0.92-1.40, P=0.230$, Figure 3D), as well as vomiting ( $\mathrm{RR}=17.11,95 \% \mathrm{CI}$ : $2.22-131.79$, $P=0.632$; Figure 3E) between the two groups.

\section{Sensitivity analysis and publication bias}

According to the Egger's test, we found no publication bias in aspects of all outcomes $(P>0.05$; Table 2$)$. Additionally, we also assessed the influence of each individual study on the combined effect size by sensitivity analysis, and the result of thrombocytopenia was inversed after removing Kim's article ${ }^{19}$ (RR $=1.11,95 \%$ CI: 0.71-1.73, $P=0.654)$. We did not perform sensitivity analysis for neutropenia and vomiting, since there were only two eligible studies.

\section{Discussion}

Currently, the curative effect of traditional chemotherapy regimens for PTCL treatment is generally dismal. ${ }^{23,24}$ Many researchers have turned to addition of etoposide on the basis of CHOP, but randomized controlled trials comparing different treatment approaches for PTCL have been very limited until now. In this meta-analysis, we included five prospective or retrospective articles, for the first time, to compare the differences of outcomes between CHOP and CHOPE for PTCL. The results revealed AEs such as anemia and thrombocytopenia in PTCL patients receiving CHOPE were more serious than in those receiving $\mathrm{CHOP}$, but the differences of $\mathrm{CR}, \mathrm{PR}$, and ORR between them were not significant.

To our knowledge, intensive chemotherapy regimens have been regarded as the first-line treatment for PTCL patients. An increasing number of research studies suggest that etoposide has been used in intensive chemotherapy regimens, such as cisplatin, etoposide, gemcitabine, and 


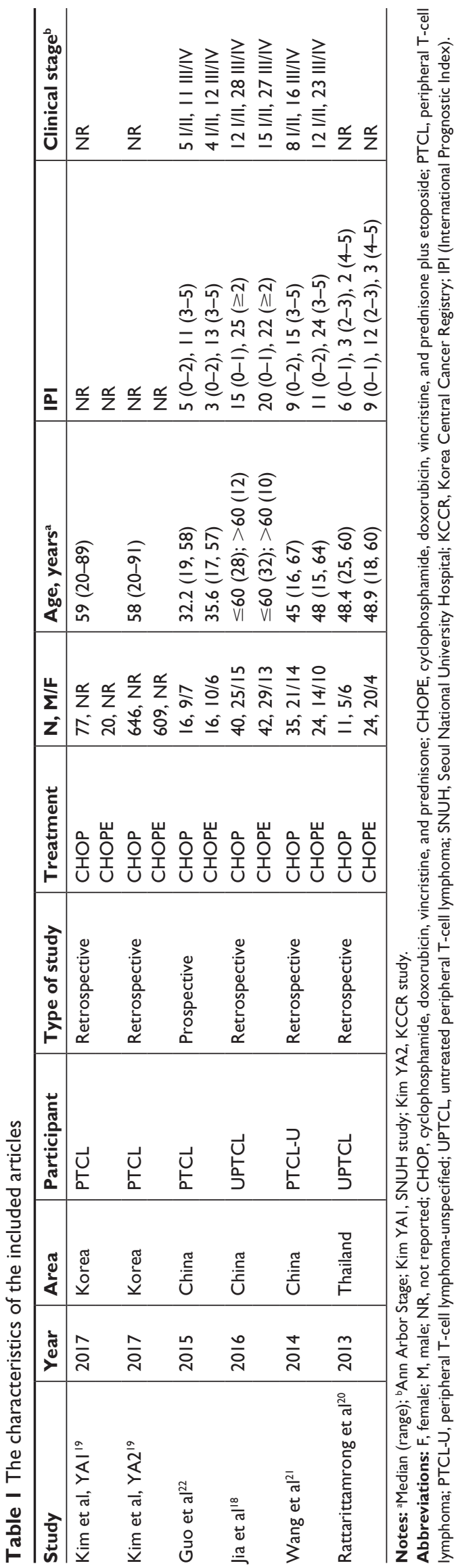

solumedrol (PEGS), and L-asparaginase and etoposide (SMILE) for PTCL. ${ }^{25,26}$ Indeed, as a standard therapy for PTCL, most patients treated with CHOP will achieve a CR ranging from $31 \%-69 \%{ }^{27}$ or a CR + PR of $48 \%{ }^{28}$ Notably, a study has demonstrated that the ORR and CR for evaluable non-Hodgkin's lymphoma patients was significantly increased in CHOPE group compared to CHOP group (ORR: $82.5 \%$ vs $62.5 \%$; CR: $52.5 \%$ vs $45 \%$ ), respectively. ${ }^{29}$ However, in this meta-analysis, we found no significant differences of CR, PR, and ORR between CHOP and CHOPE for PTCL treatment. Similarly, it has been suggested that the response and survival rates of non-Hodgkin's lymphoma patients treated with dose-escalated CHOPE are similar to those of patients treated with CHOP standard treatment. ${ }^{30}$ Our pooled result suggested that the addition of etoposide might not effectively improve the curative effect of CHOP for patients with PTCL, which was different from several single studies. One possible explanation is that patients' age is a main factor for curative effect comparison, based on an interesting study by Norbert which showed that etoposide improved response rates in younger patients with PTCL, while no significant difference was found in patients older than 60 years. ${ }^{15}$

In addition, our study revealed that the differences were significant for anemia and thrombocytopenia between CHOP and CHOPE, and publication bias analysis showed the results were reliable, suggesting the AEs were more frequently found in patients treated with CHOPE. The related AEs of PTCL during treatment are divided into five grades, and the most common AEs for grade $\geq 3$ were anemia and thrombocytopenia. ${ }^{31}$ Reportedly, although etoposide has a good antitumor action, it has major adverse effects on myelosuppression and gastrointestinal reaction, which may cause anemia and thrombocytopenia. ${ }^{32}$ Additionally, it is suggested that the addition of etoposide did not yield better treatment outcomes but was associated with more toxicity. ${ }^{19}$ Overall, the results indicated CHOPE could increase the adverse effects in comparison to CHOP, which provides the basis for the selection of chemotherapy in the future. However, to further verify the epilog, more research is still needed.

Our study made a comprehensive comparison of the efficacy and toxicity between CHOP and CHOPE for PTCL patients, and results were reliable with no significant publication bias between different studies. However, there were several limitations in this study. For example, the number of studies included in this analysis was relatively small, and were all from the Asia region, leading to unrepresentative 


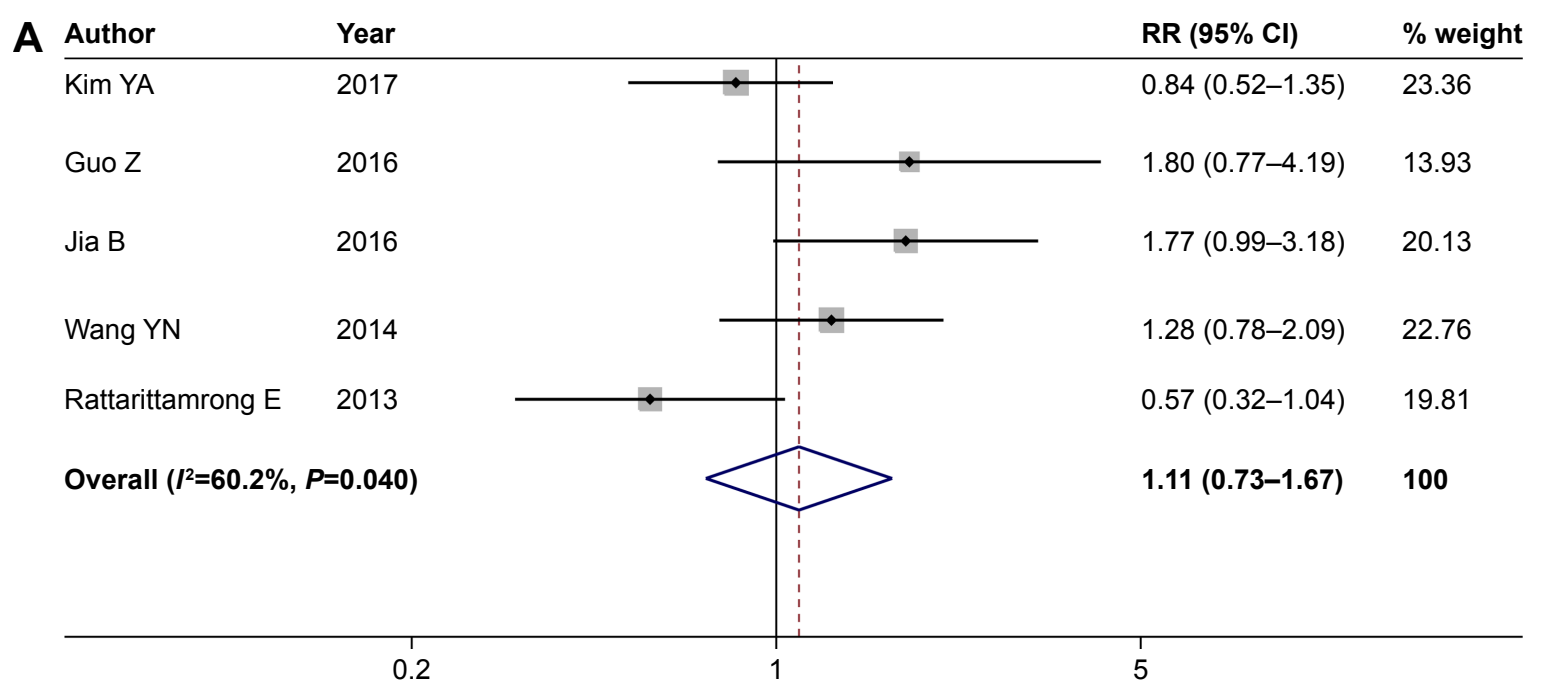

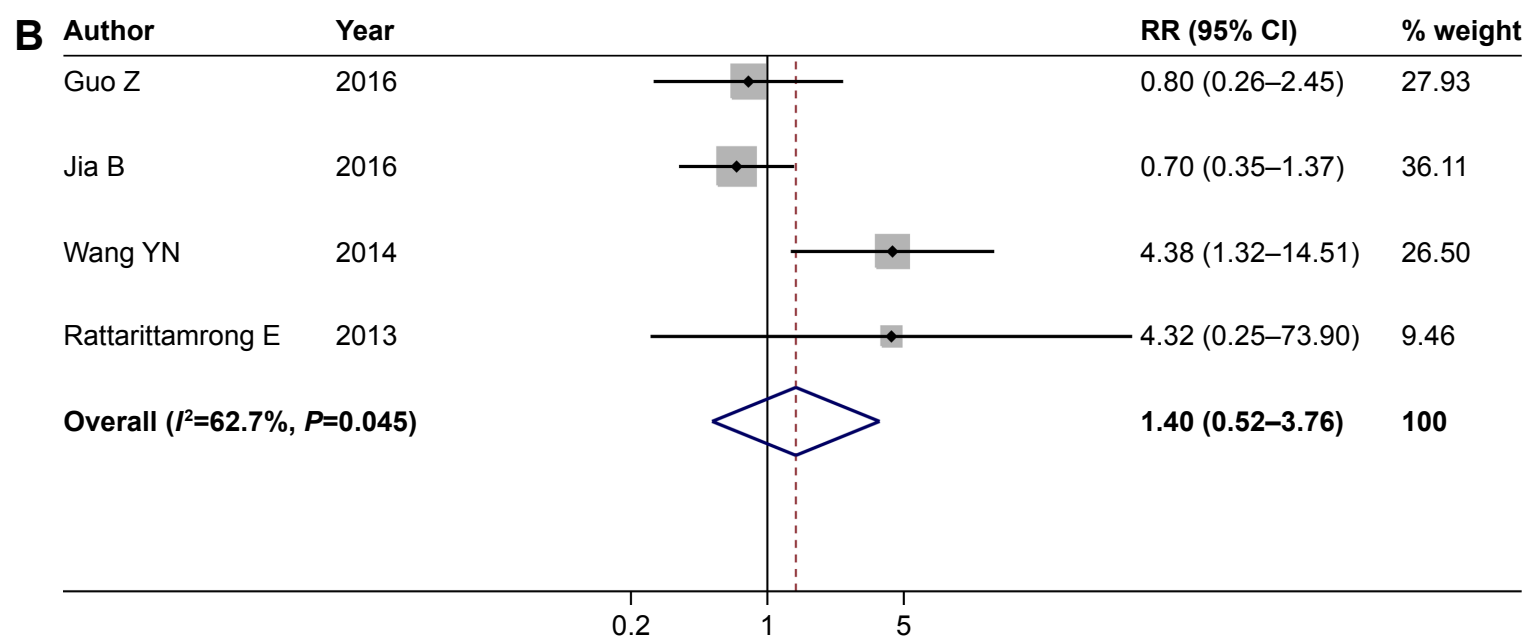

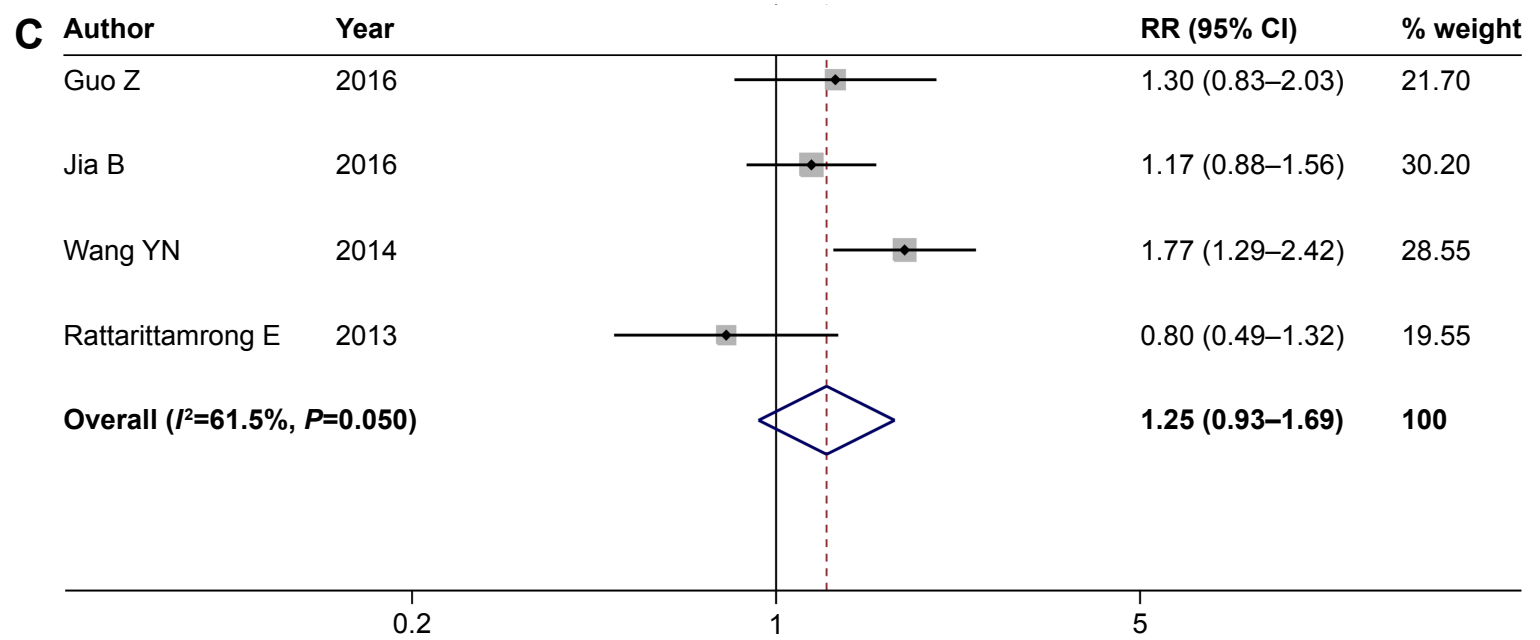

Figure 2 Meta-analysis results of CHOP and CHOPE for (A) CR, (B) PR, and (C) ORR.

Note: Weights are from random-effects analysis.

Abbreviations: CHOP, cyclophosphamide, doxorubicin, vincristine, and prednisone; CHOPE, cyclophosphamide, doxorubicin, vincristine, and prednisone plus etoposide; $\mathrm{CR}$, complete response; PR, partial response; ORR, overall response rate; RR, risk ratio. 
results. Additionally, there was heterogeneity with regards to the subjects in this study, with some researchers recruiting initial patients with PTCL, while others included initial treatment and recurrence of PTCL. Next, the included trials were mainly retrospective articles, and therefore, there was no suitable evaluation tool for quantitative evaluation.

In summary, this meta-analysis demonstrated that CHOPE significantly increased AEs, including anemia
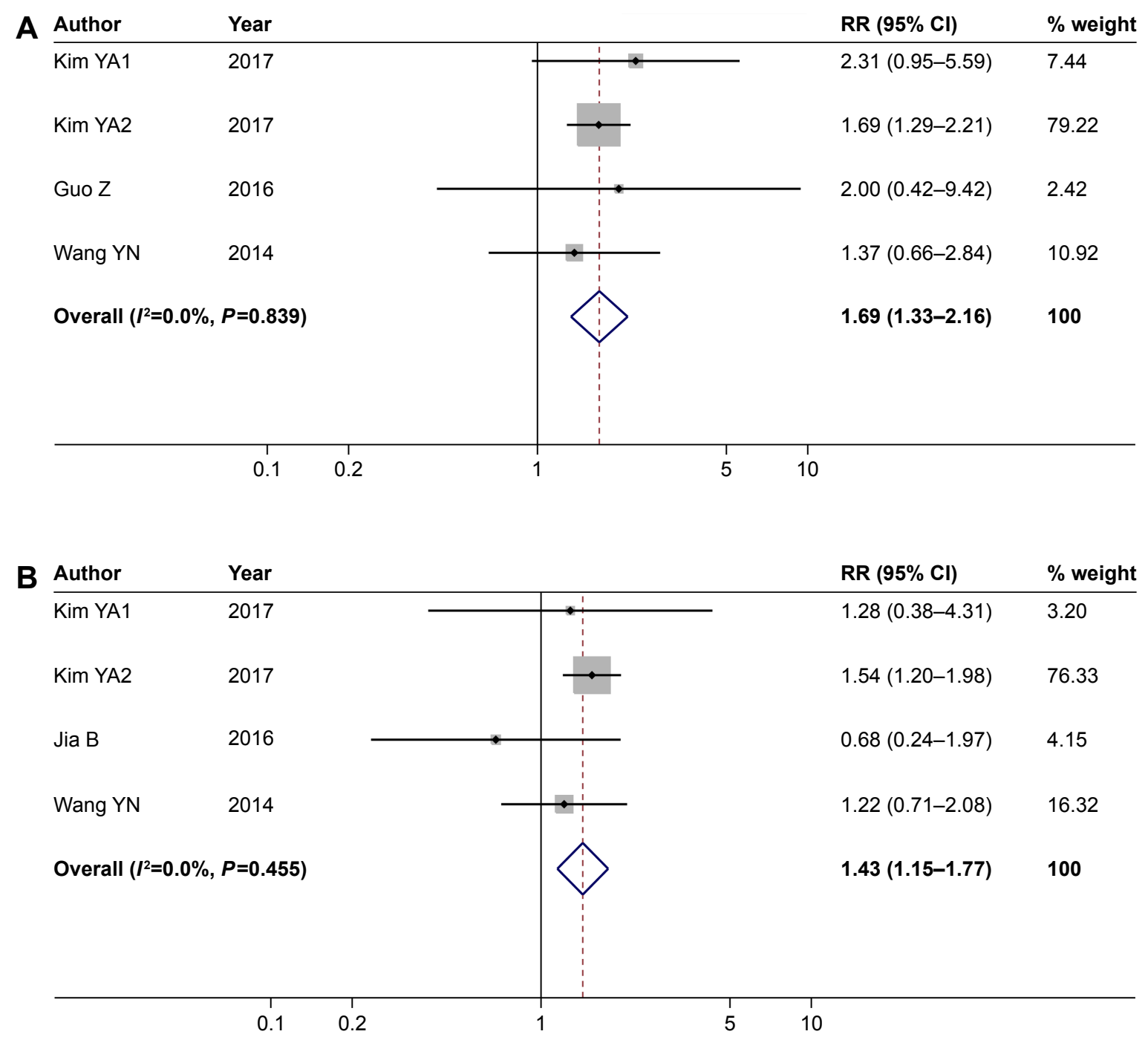

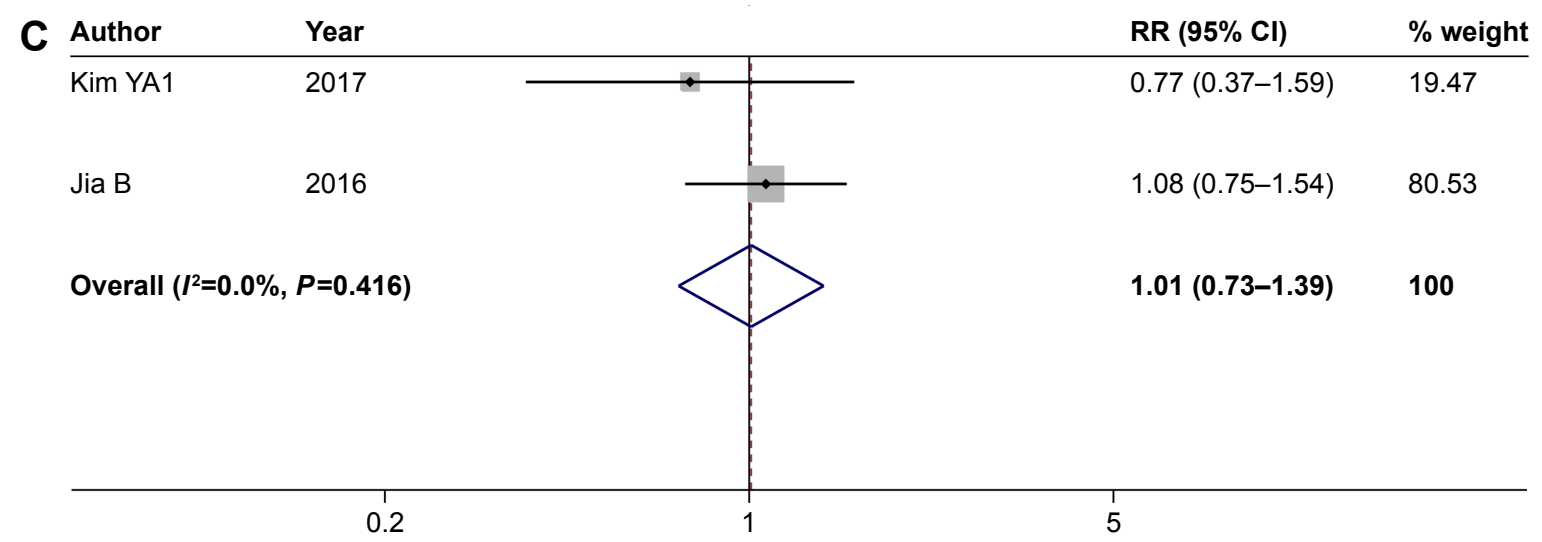

Figure 3 (Continued) 


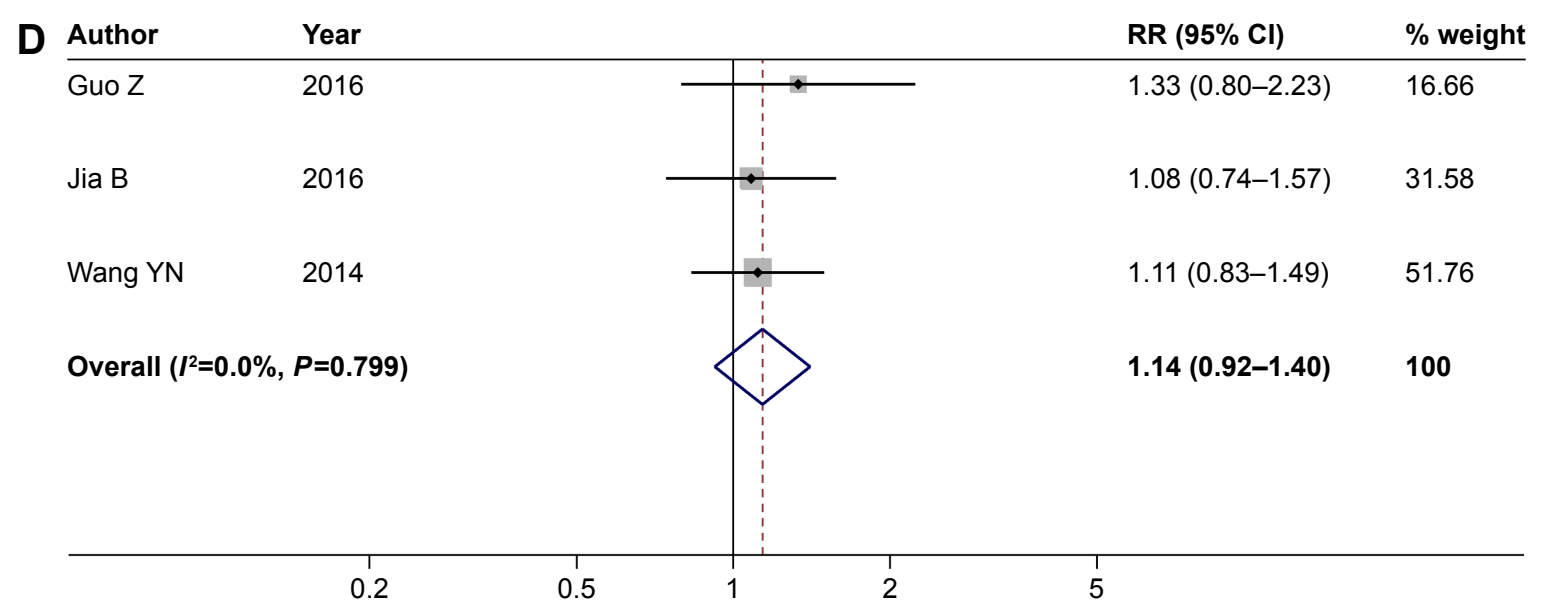

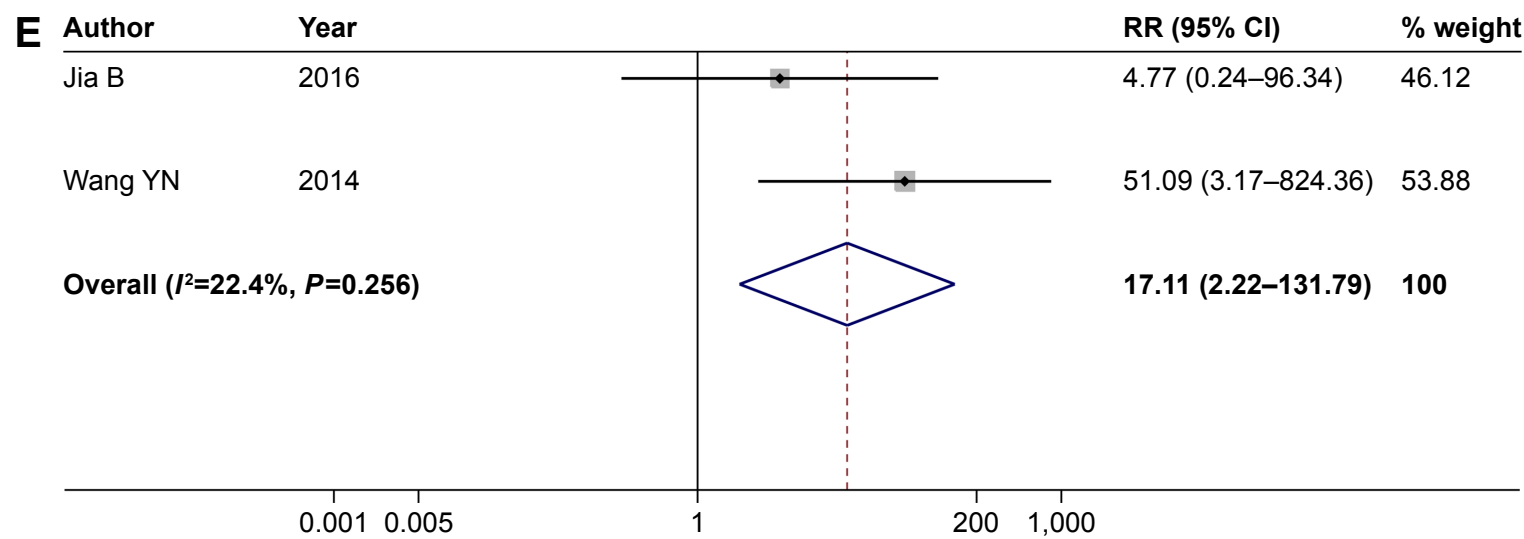

Figure 3 Meta-analysis results of CHOP and CHOPE for adverse events including (A) thrombocytopenia, (B) neutropenia, (C) anemia, (D) leukopenia, and (E) vomiting. Abbreviations: CHOP, cyclophosphamide, doxorubicin, vincristine, and prednisone; CHOPE, cyclophosphamide, doxorubicin, vincristine, and prednisone plus etoposide; $\mathrm{RR}$, risk ratio.

and thrombocytopenia in patients with PTCL, and the therapeutic effects of CHOP and CHOPE for treating PTCL were not significant with regard to $C R$, PR, and ORR. However, to further verify the results, a large number of clinical randomized controlled studies are still needed.

Table 2 Sensitivity analysis and publication bias

\begin{tabular}{|c|c|c|c|}
\hline Outcomes & $\mathbf{N}$ & $\begin{array}{l}\text { Sensitivity analysis, } \\
\text { RR }(95 \% \mathrm{Cl})\end{array}$ & $\begin{array}{l}\text { Egger, } \\
P\end{array}$ \\
\hline $\mathrm{CR}$ & 5 & $0.98(0.64-1.50)-1.27(0.89-1.83)$ & 0.574 \\
\hline PR & 4 & $0.78(0.44-1.37)-2.10(0.57-7.75)$ & 0.335 \\
\hline ORR & 4 & I.II (0.88-I.4I)-I.39 (I.07-I.82) & 0.504 \\
\hline Neutropenia & 2 & - & - \\
\hline Anemia & 4 & $1.65(1.29-2.12)-1.74$ (1.35-2.25) & 0.720 \\
\hline Thrombocytopenia & 4 & $1.11(0.71-1.73)-1.47(1.18-1.84)$ & 0.171 \\
\hline Leukopenia & 3 & $1.10(0.87-1.39)-1.16(0.90-1.50)$ & 0.415 \\
\hline Vomiting & 2 & - & - \\
\hline
\end{tabular}

Abbreviations: $C R$, complete response; PR, partial response; ORR, overall response rate; $\mathrm{RR}$, risk ratio.

\section{Acknowledgments}

The study was funded by the State Administration of Traditional Chinese Medicine, National Chinese Medicine Clinical Research Base Research special (2015H03); the Zhejiang Natural Science Foundation Project (LQ18H08000); the medical and health general research program of Zhejiang Province (2016KYA151); and the Chinese Medicine Research Fund Project of Zhejiang Province (2016ZA080).

\section{Disclosure}

The authors report no conflicts of interest in this work.

\section{References}

1. Yang YT, Tai CJ, Chen C, et al. Highly diverse efficacy of salvage treatment regimens for relapsed or refractory peripheral T-cell lymphoma: a systematic review. PLoS One. 2016;11(10):e0161811.

2. Park S, Ko YH. Peripheral T cell lymphoma in Asia. Int $J$ Hematol. 2014;99(3):227-239

3. Armitage JO. The aggressive peripheral T-cell lymphomas: 2012 update on diagnosis, risk stratification, and management. Am J Hematol. 2012;87(5):511-519. 
4. Vose J, Armitage J, Weisenburger D. International T-Cell Lymphoma Project. International peripheral T-cell and natural killer/T-cell lymphoma study: pathology findings and clinical outcomes. J Clin Oncol. 2008; 26(25):4124-4130.

5. Zelenetz AD, Abramson JS, Advani RH, et al. Nccn clinical practice guidelines in oncology: non-Hodgkin's lymphomas. J Natl Compr Canc Netw. 2010;8(3):288-334.

6. Mak V, Hamm J, Chhanabhai M, et al. Survival of patients with peripheral T-cell lymphoma after first relapse or progression: spectrum of disease and rare long-term survivors. J Clin Oncol. 2013;31(16): 1970-1976.

7. Coiffier B, Pro B, Prince HM, et al. Results from a pivotal, open-label, phase II study of romidepsin in relapsed or refractory peripheral T-cell lymphoma after prior systemic therapy. J Clin Oncol. 2012;30(6): 631-636.

8. Foss F, Advani R, Duvic M, et al. A phase II trial of belinostat (pxd101) in patients with relapsed or refractory peripheral or cutaneous T-cell lymphoma. Br J Haematol. 2015;168(6):811-819.

9. Pro B, Advani R, Brice P, et al. Brentuximab vedotin (sgn-35) in patients with relapsed or refractory systemic anaplastic large-cell lymphoma: results of a phase II study. J Clin Oncol. 2012;30(18):2190-2196.

10. O'Connor OA, Pro B, Pinter-Brown L, et al. Pralatrexate in patients with relapsed or refractory peripheral T-cell lymphoma: results from the pivotal PROPEL study. J Clin Oncol. 2011;29(9):1182-1189.

11. Thakur DS. Topoisomerase II inhibitors in cancer treatment. Ijpsn. 2011;3(4):1173-1181.

12. Schonn I, Hennesen J, Dartsch DC. Cellular responses to etoposide: cell death despite cell cycle arrest and repair of DNA damage. Apoptosis. 2010;15(2):162-172.

13. Pfreundschuh M, Trümper L, Kloess M, et al. Two-weekly or 3-weekly CHOP chemotherapy with or without etoposide for the treatment of elderly patients with aggressive lymphomas: results of the NHL-B2 trial of the DSHNHL. Blood. 2004;104(3):634-641.

14. Peng YL, Huang HQ, Lin XB, et al. [Clinical outcomes of patients with peripheral T-cell lymphoma (ptcl) treated by epoch regimen]. Ai Zheng. 2004;23(8):943-946.

15. Schmitz N, Trümper L, Ziepert M, et al. Treatment and prognosis of mature T-cell and NK-cell lymphoma: an analysis of patients with T-cell lymphoma treated in studies of the German high-grade non-Hodgkin lymphoma study group. Blood. 2010;116(18):3418-3425.

16. Nickelsen M, Ziepert M, Zeynalova S, et al. High-dose chop plus etoposide (Megachoep) in T-cell lymphoma: a comparative analysis of patients treated within trials of the German high-grade non-Hodgkin lymphoma Study Group (dshnhl). Ann Oncol. 2009;20(12):1977-1984.

17. Higgins JP, Thompson SG, Deeks JJ, Altman DG. Measuring inconsistency in meta-analyses. BMJ. 2003;327(7414):557-560.

18. Jia B, Hu S, Yang J, et al. Comparison of gemcitabin, cisplatin, and dexamethasone (gdp), CHOP, and CHOPE in the first-line treatment of peripheral T-cell lymphomas. Hematology. 2016;21(9):536-541.
19. Kim YA, Byun JM, Park K, et al. Redefining the role of etoposide in firstline treatment of peripheral T-cell lymphoma. Blood Advances. 2017; 1(24):2138-2146.

20. Rattarittamrong E, Norasetthada L, Tantiworawit A, Chai-Adisaksopha C, Nawarawong W. CHOEP-21 chemotherapy for newly diagnosed nodal peripheral T-cell lymphomas (ptcls) in Maharaj Nakorn Chiang Mai Hospital. J Med Assoc Thai. 2013;96(11):1416.

21. Wang YN, Liu WD, Ma SD, et al. Historical controlled clinical study of the first line treatment for peripheral T-cell lymphomas-unspecified with epoch and CHOP regimen. J Modern Oncology. 2014;22:420-422. Chinese.

22. Guo Z, Chen HR, Luo JX, et al. Clinical Controlled Trial of Two Firstline Chemotherapy Treatment for Peripheral T Cell Lymphoma. The Practical Journal of Cancer. 2016;31(7):1105-1107.

23. Zelenetz AD, Gordon LI, Wierda WG, et al. Non-Hodgkin's lymphomas, version 4.2014. J Natl Compr Canc Netw. 2014;12(9):1282-1303.

24. Shibata Y, Hara T, Kasahara S. CHOP or THP-COP regimens in the treatment of newly diagnosed peripheral T-cell lymphoma, not otherwise specified: a comparison of doxorubicin and pirarubicin. Hematological Oncology. 2015;73(4):252-256.

25. Mahadevan D, Unger JM, Spier CM, et al. Phase 2 trial of combined cisplatin, etoposide, gemcitabine, and methylprednisolone (pegs) in peripheral t-cell non-hodgkin lymphoma. Cancer. 2013;119(2):371-379.

26. Yamaguchi M, Kwong YL, Kim WS, et al. Phase II study of SMILE chemotherapy for newly diagnosed stage IV, relapsed, or refractory extranodal natural killer (NK)/T-cell lymphoma, nasal type: the NK-Cell Tumor Study Group study. J Clin Oncol. 2011;29(33):4410-4416.

27. Akagi T, Takahashi N, Yamaguchi K, et al. Comparison of long-term clinical outcomes of CHOP chemotherapy between Japanese patients with nodal peripheral T-cell lymphomas and those with diffuse large B-cell lymphoma in the study Group of the Tohoku hematology forum. J Clin Exp Hematopathol. 2011;51(1):29-35.

28. Mercadal S, Briones J, Xicoy B, et al. Intensive chemotherapy (highdose chop/eshap regimen) followed by autologous stem-cell transplantation in previously untreated patients with peripheral T-cell lymphoma. Ann Oncol. 2008;19(5):958-963.

29. Lu W. Preliminary study of CHOPE treatment for patients with advanced non-Hodgkin's lymphoma (NHL). Chinese Journal of Clinical Oncology. 2007;34(3):151-153. Chinese.

30. Bartlett NL, Petroni GR, Parker BA, et al. Dose-escalated cyclophosphamide, doxorubicin, vincristine, prednisone, and etoposide (CHOPE) chemotherapy for patients with diffuse lymphoma: Cancer and leukemia group B studies 8852 and 8854. Cancer. 2001;92(2):207.

31. Maeda Y, Nishimori H, Yoshida I, et al. Dose-adjusted epoch chemotherapy for untreated peripheral T-cell lymphomas: a multicenter phase II trial of West-JHOG PTCL0707. Haematologica. 2017;102(12):2097-2103.

32. Suzuki K, Nakamura T, Kato N, et al. [Preliminary report on the effect of etoposide or etoposide and CDDP on recurrent prostate cancer]. Hinyokika Kiyo. 1991;37(11):1499-1503.
OncoTargets and Therapy

\section{Publish your work in this journal}

OncoTargets and Therapy is an international, peer-reviewed, open access journal focusing on the pathological basis of all cancers, potential targets for therapy and treatment protocols employed to improve the management of cancer patients. The journal also focuses on the impact of management programs and new therapeutic agents and protocols on
Dovepress

patient perspectives such as quality of life, adherence and satisfaction. The manuscript management system is completely online and includes a very quick and fair peer-review system, which is all easy to use. Visit http://www.dovepress.com/testimonials.php to read real quotes from published authors. 